\title{
Isomorphisms between quantum generalized Weyl algebras
}

\author{
Lionel Richard* and Andrea Solotar ${ }^{\dagger}$
}

November 10, 2018

\begin{abstract}
We study isomorphisms between generalized Weyl algebras, giving a complete answer to the quantum case of this problem for $R=k[h]$.
\end{abstract}

\section{Introduction}

Generalized Weyl algebras (GWA for short), have been defined by V. Bavula in [3] and widely studied by himself and collaborators in a series of papers (see for example [3] , 5], 6]) from the point of view of ring theory.

This kind of algebras has also been studied by T. J. Hodges [11] with the name of Non commutative deformations of type A-Kleinian singularities.

Examples of GWA are, $n$-th Weyl algebras, $\mathcal{U}\left(\mathfrak{s l}_{2}\right)$, primitive quotients of $\mathcal{U}\left(\mathfrak{s l}_{2}\right)$, its quantized versions and also the subalgebras of invariants of these algebras under the action of finite cyclic subgroups of automorphisms.

A GWA over a field $k$ is defined as follows: Let $R$ be an associative noetherian $k$-algebra which is an integral domain; $A=R(\sigma, a)$, with $\sigma \in \operatorname{Aut}(R)$ and $a$ a nonzero central element of $R$, is the $k$-algebra generated over $R$ by two generators $x$ and $y$ with relations :

$$
\begin{gathered}
x r=\sigma(r) x, \forall r \in R \\
y r=\sigma^{-1}(r) y, \forall r \in R \\
x y=\sigma(a), \\
y x=a .
\end{gathered}
$$

The algebra $A$ is a noetherian domain (see [3]).

Concerning the problem of isomorphism between two GWA $A=R(\sigma, a)$ and $A^{\prime}=R\left(\sigma, a^{\prime}\right)$ raised in [1], it is known after [5] that:

\footnotetext{
*Institut Girard Desargues, Université Lyon 1. F-69622 Villeurbanne Cedex - France. richard@igd.univ-lyon1.fr

${ }^{\dagger}$ Dto. de Matemática, Facultad de Cs. Exactas y Naturales. Universidad de Buenos Aires. Ciudad Universitaria Pab I. (1428), Buenos Aires - Argentina. asolotar@dm.uba.ar

${ }^{0}$ Research partially supported by UBACYT TW62, PICS-CNRS 1514, FUNDACIÓN ANTORCHAS, ANPCYT, PICT 03-08280 and COOPERACIÓN INTERNACIONAL-CONICET. The second author is a research member of CONICET (Argentina).
} 
- If $R=k[h]$ and $\sigma(h)=h-1$, then $A \cong A^{\prime} \Longleftrightarrow \exists \eta \in k^{*}, \beta \in k$ such that $a(h)=\eta a^{\prime}(h-\beta)$.

- If $R=k\left[h, h^{-1}\right]$ and $\sigma(h)=q h, q \in k^{*}$ not a root of unity, then $A \cong A^{\prime} \Longleftrightarrow$ $\exists \eta, \beta \in k^{*}, m \in \mathbb{Z}$ and $\epsilon \in\{1,-1\}$ such that $a^{\prime}(h)=\eta h^{m} a^{\prime}\left(\beta h^{\epsilon}\right)$.

The main result of this paper gives an answer to the quantum case of this problem for $R=k[h]$. Namely we obtain the following Theorem.

Theorem 1.1 Set $q \in k^{*}$ not a root of unity, $a_{1}, a_{2} \in k[h]$ non-constant polynomials and $\sigma(h)=q h$. Then there is an isomorphism $\Phi$ from the quantum $G W A$ $A_{q}\left(a_{1}\right)=k[h]\left(\sigma, a_{1}\right)$ onto $A_{q}\left(a_{2}\right)=k[h]\left(\sigma, a_{2}\right)$ if and only if there exist $\alpha, \rho \in k^{*}$ such that $a_{2}(h)=\rho a_{1}(\alpha h)$.

The above theorem covers for example the cases of the quantum Weyl algebra $A_{1}^{q}$, $k$-generated by variables $x, y$ such that $x y-q y x=1$, and the algebras $D_{q, \lambda}$ defined in [2], that is, the quotients of the ad-locally finite elements $F_{q}$ of $\mathcal{U}_{q}\left(\mathfrak{s l}_{2}\right)$ by the ideal generated by $(\Omega-\lambda)$. The algebra $D_{q, \lambda}$ verifies that $\operatorname{Aut}\left(D_{q, \lambda}\right) \cong \operatorname{Aut}\left(F_{q}\right)$. In this case, our result says that $D_{q, \lambda} \cong D_{q, \lambda^{\prime}}$ if and only if $\lambda^{2}=\lambda^{\prime 2}$.

We also solve the problem of isomorphisms of quantum Smith algebras, generalizing Theorem 5.4 of [5]. This allows us to treat some cases of Witten's deformations of $\mathcal{U}\left(\mathfrak{s l}_{2}\right)$ and of conformal $\mathfrak{s l}_{2}$ algebras (see [7]).

Finally we give a necessary condition for Morita equivalence of GWA for $R=k[h]$. This problem has been solved by Hodges [10] for $\sigma(h)=h-1$ and $\operatorname{deg}(a)=2$. Here we look at the quantum case for arbitrary $a$.

The article is organized as follows:

In section 2 we recall the canonical forms of GWA, for $R=k[h]$, separating the classical case from the quantum one by means of two invariants defined by Alev and Dumas in [1. We also rephrase Theorem 3.3 of [5] (isomorphisms between classical GWA) in terms of the roots of the defining polynomials of the algebras. We then prove a technical lemma and our main result.

In section 3 we give a necessary condition for Morita equivalence.

We will work over an algebraically closed field $k$ of characteristic zero and all algebras will be $k$-algebras. By "domain" we shall mean an integral ring, not necessarily commutative.

We consider GWA of degree one : $A=k[h](\sigma, a)$, with $\sigma \in \operatorname{Aut}(k[h])$ and $a \in$ $k[h] \backslash\{0\}$, is the $k$-algebra generated over $k[h]$ by two generators $x$ and $y$ with relations :

$$
\begin{gathered}
x h=\sigma(h) x, \\
y h=\sigma^{-1}(h) y, \\
x y=\sigma(a), \\
y x=a .
\end{gathered}
$$

Anywhere in this text "Morita equivalence" means " $k$-linear Morita equivalence", and morphisms are morphisms of $k$-algebras. 
We will suppose that $a$ is not constant, because if $a=a_{0} \in k^{*}$, then $A$ is the Ore extension $k[h]\left(x^{ \pm 1} ; \sigma, 0\right)$ whose properties have been extensively studied.

We want to thank François Dumas for bringing to our attention the article 2.

\section{Isomorphism and rational equivalence of GWA.}

\subsection{General results.}

Let us first present "canonical" forms of GWA of degree one and of their fraction fields.

Proposition 2.1.1 Let $A=k[h](\sigma, a)$ be a GWA of degree one. Then necessarily $\sigma(h)=q h-h_{0}$ with $q \in k^{*}$ and $h_{0} \in k$, and we have one of the three following alternatives :

1. $\sigma=\mathrm{id}$, i.e. $\left(q, h_{0}\right)=(1,0)$, then $A$ is commutative, and $\operatorname{Frac}(A)$ is the (commutative) field of rational functions $k(h, x)$;

2. $q=1$ and $h_{0} \neq 0$, then $A$ is isomorphic to $k\left[h^{\prime}\right]\left(\sigma_{c l}, a^{\prime}\right)$, with $\sigma_{c l}\left(h^{\prime}\right)=h^{\prime}-1$ and $a^{\prime}\left(h^{\prime}\right)=a\left(h_{0} h^{\prime}\right)$, and $\operatorname{Frac}(A)$ is the first Weyl division algebra $\mathcal{D}_{1}(k)$ ("classical" case) ;

3. $q \neq 1$, then $A$ is isomorphic to $k\left[h^{\prime}\right]\left(\sigma_{q}, a^{\prime}\right)$, with $\sigma_{q}\left(h^{\prime}\right)=q h^{\prime}$ and $a^{\prime}\left(h^{\prime}\right)=$ $a\left(h^{\prime}-h_{0} /(1-q)\right)$, and $\operatorname{Frac}(A)$ is the quantum skew-field $k_{q}\left(x, h^{\prime}\right)$ ("quantum" case).

Proof. The first case is obvious. In the second one the "canonical" form is obtained by setting $h^{\prime}=h_{0}^{-1} h$, and the division algebra $\operatorname{Frac}(A)$ is the fraction field of the $k$-algebra generated by $x$ and $h$ because $y=a(h) x^{-1}$. It is also the fraction field of the $k$-algebra generated by $x$ and $y^{\prime}=-h_{0}^{-1} h x^{-1}$, and one easily checks that $\left[x, y^{\prime}\right]=1$, so this $k$-algebra is nothing but the first Weyl algebra (because we are in characteristic zero, any homomorphic image of $A_{1}(k)$ is an isomorphic image).

Suppose now that $q \neq 1$, the isomorphism $\sigma_{q}$ is obtained by setting $h^{\prime}=h+h_{0} /(1-$ $q$ ), and again because $y=a(h) x^{-1}$, we see that $\operatorname{Frac}(A)$ is the fraction field of the $k$-algebra generated by $x$ and $h$, or by $x$ and $y^{\prime}=h x-x h=\left((1-q) h+h_{0}\right) x$. One can then easily check that $x y^{\prime}=q y^{\prime} x$, and these two generators are algebraically independent over $k$.

The following invariants, introduced by Alev and Dumas in [1, separate these three cases.

Definition 2.1.2 For any associative $k$-algebra A, one notes :

- $G(A)=\left(A^{*}\right)^{\prime} \cap k^{*}$ the trace on $k^{*}$ of the derived group from the multiplicative group $A^{*}$ of units in $A$; 
- $E(A)=[A, A] \cap k$ the trace on $k$ of the derived Lie algebra from $A$.

Remark. The space $E(A)$ can only be $k$ or $\{0\}$, according to the existence or not of two elements $x, y \in A$ such that $[x, y]=1$.

The following proposition separates Weyl division algebras and quantum skewfields.

Proposition 2.1.3 $\quad$ 1. The first Weyl division algebra $\mathcal{D}_{1}(k)$ satisfies :

$$
G\left(\mathcal{D}_{1}(k)\right)=\{1\}, \quad \text { and } E\left(\mathcal{D}_{1}(k)\right)=k .
$$

2. Set $q \in k^{*}$, then the quantum skew-field $k_{q}(u, v)$ satisfies :

$$
E\left(k_{q}(u, v)\right)=\{0\}, \quad \text { and } G\left(k_{q}(u, v)\right)=\langle q\rangle,
$$

the multiplicative subgroup of $k^{*}$ generated by $q$.

Proof. These are particular cases of proposition 3.9 and Théorème 3.10 of [1].

Corollary 2.1.4 The Weyl algebra $A_{1}(k)$ never imbeds in the fraction field of a noncommutative quantum plane $k_{q}[u, v]$, and vice-versa.

Proof. The proof is direct from proposition 2.1.3

Remark. The GWA $k[h](\sigma, a(h))$ is isomorphic to $k[h]\left(\sigma^{-1}, a(\sigma(h))\right)$ by $(x, y) \mapsto$ $(y, x)$, so $k[h]\left(\sigma_{q}, a(h)\right) \cong k[h]\left(\sigma_{q^{-1}}, a(q h)\right)$. Because two isomorphic GWA must be rationally equivalent, and thanks to propositions 2.1.1 and 2.1.3 we may consider the isomorphism problem for fixed $\sigma=\sigma_{c l}$ or $\sigma=\sigma_{q}, q \in k^{*}$.

In the classical case, the isomorphism problem is completely solved by Bavula and Jordan in [5] in the following way.

Theorem 2.1.5 Let $A=k[h]\left(\sigma_{c l}, a_{1}\right)$ and $B=k[h]\left(\sigma_{c l}, a_{2}\right)$ be two classical $G W A$, with $\sigma_{c l}(h)=h-1$, and $a_{1}, a_{2} \in k[h]$. Then $A$ and $B$ are isomorphic if and only if $a_{2}(h)=\rho a_{1}(\epsilon h+\alpha)$, for some $\rho \in k^{*}, \alpha \in k$ and $\epsilon \in\{-1,1\}$.

Proof. This is Theorem 3.3 of [5].

Let us rephrase this result in terms of the roots of the polynomials $a_{1}$ and $a_{2}$ in the case where these are non-constant polynomials.

Corollary 2.1.6 Let $A=k[h]\left(\sigma_{c l}, a_{1}\right)$ and $B=k[h]\left(\sigma_{c l}, a_{2}\right)$ be two classical $G W A$, with $\sigma_{c l}(h)=h-1$, and $a_{1}, a_{2} \in k[h] \backslash k$. Write $a_{1}=\prod_{i=1}^{n}\left(h-\alpha_{i}\right)$ and $a_{2}=\prod_{i=1}^{m}\left(h-\beta_{i}\right)$ Then the following assertions are equivalent:

1. $A$ and $B$ are isomorphic;

2. $n=m$, and there exists $\tau \in \mathcal{S}_{n}$ such that for all $1 \leq i, j \leq n$, one has $\left(\alpha_{i}-\alpha_{j}\right)^{2}=\left(\beta_{\tau(i)}-\beta_{\tau(j)}\right)^{2}$ 
3. $n=m$, and there exist $\tau \in \mathcal{S}_{n}$ and $\epsilon \in\{-1,1\}$ such that for all $1 \leq i \leq n$, one has $\alpha_{i}-\alpha_{1}=\epsilon\left(\beta_{\tau(i)}-\beta_{\tau(1)}\right)$.

Proof. It is clear that by Theorem 2.1.5 the first point implies points 2 and 3. Clearly point 3 implies point 2 . So we only have to check that if $a_{1}$ and $a_{2}$ satisfy the second point, then we have $a_{2}(h)=\rho a_{1}(\epsilon h+\alpha)$, with $\rho \in k^{*}, \alpha \in k$ and $\epsilon \in\{-1,1\}$.

Suppose first that both $a_{1}$ and $a_{2}$ only have simple roots. By hypothesis, for each $(i, j) \in\{1, \ldots, n\}^{2}$ with $i \neq j$ there is $\epsilon_{i, j} \in\{-1,1\}$ such that $\alpha_{i}-\alpha_{j}=$ $\epsilon_{i, j}\left(\beta_{\tau(i)}-\beta_{\tau(j)}\right)$. By Theorem 2.1.5 $A$ is isomorphic to $k[h]\left(\sigma_{c l}, a_{1}(-h)\right)$, and the roots of $a_{1}(-h)$ are $-\alpha_{1}, \ldots,-\alpha_{n}$. So without loss of generality one may suppose that $\epsilon_{1,2}=1$. By reordering the roots of $a_{2}$, one may also suppose that $\tau=\mathrm{Id}$. Let $\beta=\beta_{1}-\alpha_{1}$. We show now that the $\beta_{i}-\beta, 1 \leq i \leq n$ are the roots of $a_{1}$. Since $a_{1}$ and $a_{2}$ have exactly $n$ simple roots, this will show that $a_{2}(h)=\rho a_{1}(h+\beta)$ for some $\rho \in k^{*}$.

By definition $\beta_{1}-\beta=\alpha_{1}$ is a root of $a_{1}$, and $\beta_{2}-\beta=\beta_{2}-\left(\beta_{1}-\alpha_{1}\right)=$ $\epsilon_{1,2}\left(\alpha_{2}-\alpha_{1}\right)+\alpha_{1}=\alpha_{2}$ is a root of $a_{1}$ too. Now for any $i \geq 3$, we have

$$
\alpha_{i}=\alpha_{1}-\left(\alpha_{1}-\alpha_{i}\right)=\beta_{1}-\beta-\epsilon_{1, i}\left(\beta_{1}-\beta_{i}\right)=\epsilon_{1, i} \beta_{i}-\beta+\left(1-\epsilon_{1, i}\right) \beta_{1} .
$$

In an analogous way we show that $\alpha_{i}=\epsilon_{2,1} \beta_{i}-\beta+\left(1-\epsilon_{2, i}\right) \beta_{2}$. In particular

$$
\left(1-\epsilon_{1, i}\right) \beta_{1}+\epsilon_{1, i} \beta_{i}=\left(1-\epsilon_{2, i}\right) \beta_{2}+\epsilon_{2, i} \beta_{i} .
$$

If $\epsilon_{1, i}=-1$, then $2 \beta_{1}-\beta_{i}=\left(1-\epsilon_{2, i}\right) \beta_{2}+\epsilon_{2, i} \beta_{i}$. This last expression equals $\beta_{i}$ when $\epsilon_{2, i}=1$ and $2 \beta_{2}-\beta_{i}$ when $\epsilon_{2, i}=-1$. In the first case, we obtain $\beta_{1}=\beta_{i}$, and in the second one, $2 \beta_{1}=2 \beta_{2}$. Both conditions contradict our hypothesis concerning the roots of $a_{2}$.

If $\epsilon_{1, i}=1$, then $\alpha_{i}=\beta_{i}-\beta$ is a root of $a_{1}$, and we are done.

In the general case, point 2 implies that if $\alpha_{i}$ has multiplicity $m$ in $a_{1}$, then $\beta_{\tau(i)}$ has multiplicity $m$ too in $a_{2}$. Now the proof follows from the case where all the roots are different.

\subsection{The quantum case.}

The isomorphism problem was solved too in [5] for GWA of type $k\left[h^{ \pm 1}\right]\left(\sigma_{q}, a\right)$ with $a \in k\left[h^{ \pm 1}\right]$.

Theorem 2.2.1 Let $A=k\left[h^{ \pm 1}\right]\left(\sigma_{q}, a_{1}\right)$ and $B=k\left[h^{ \pm 1}\right]\left(\sigma_{q}, a_{2}\right)$ be two quantum $G W A$, with $\sigma_{q}(h)=q h, q \in k^{*}$, and $a_{1}, a_{2} \in k\left[h^{ \pm 1}\right]$. Then $A$ and $B$ are isomorphic if and only if $a_{2}(h)=\rho h^{m} a_{1}\left(\alpha h^{\epsilon}\right)$, for some $\rho, \alpha \in k^{*}, m \in \mathbb{Z}$, and $\epsilon \in\{-1,1\}$.

Proof. This is Theorem 5.2 of [5].

Let us now consider the quantum GWA $k[h]\left(\sigma_{q}, a\right)$ with $a \in k[h]$. The preceding result cannot be used to resolve this case, because its proof relies on the fact that 
$h$ is invertible in $k\left[h^{ \pm 1}\right]$. We will see in lemma 2.2 .3 that $h$ generates (multiplicatively) the set of normalizing elements in $k[h]\left(\sigma_{q}, a\right)$.

Examples • For $a \in k^{*}$ we obtain the following localization of the quantum plane $A_{q}(a)=k_{q}\left[x^{ \pm 1}, h\right]$.

- For $\operatorname{deg}(a)=1$ the algebra $A_{q}(a)$ is generated by $x$ and $y$, and one gets Manin's quantum plane $k_{q}[x, y]$ for $a=h$, and the quantum Weyl algebra $A_{1}^{q}(k)$ for $a=h-h_{0}$, with $h_{0} \in k^{*}$.

- For $\operatorname{deg}(a)=2$ and $a(0) \neq 0$, thanks to the proposition below one can suppose without loss of generality that $a(h)=-q^{-1} /(q-1) h^{2}+q^{-1} \lambda(q-1) / q^{\prime 2} h-1 /(q-1)$, for some $\lambda \in k$, with $q^{\prime} \in k^{*}$ such that $q^{4}=q$. Then the algebra $A_{q}(a)$ is a particular subalgebra of the primitive quotient $B_{q^{\prime}, \lambda}$ of the quantum enveloping algebra $U_{q^{\prime}}(\mathfrak{s l}(2))$. More precisely (see [2] for details), $A_{q}(a)=D_{q^{\prime}, \lambda}$ is the canonical image in $B_{q^{\prime}, \lambda}$ of ad-locally finite elements in $U_{q^{\prime}}(\mathfrak{s l}(2))$.

Note that the (non-Laurent) polynomial version of the condition appearing in Theorem 2.2.1 is obviously sufficient to obtain isomorphic GWA.

Proposition 2.2.2 Set $q \in k^{*}$, and let $A_{q}\left(a_{1}\right)=k[h]\left(\sigma_{q}, a_{1}\right)$ and $A_{q}\left(a_{2}\right)=$ $k[h]\left(\sigma_{q}, a_{2}\right)$ be two quantum $G W A$ of degree one, with $\sigma_{q}(h)=q h$, and $a_{1}, a_{2} \in k[h]$ such that $a_{2}(h)=\rho a_{1}(\beta h)$, with $\rho, \beta \in k^{*}$. Then $A_{q}\left(a_{1}\right)$ and $A_{q}\left(a_{2}\right)$ are isomorphic.

Proof. The isomorphism is defined by $h \mapsto \beta h, x \mapsto \rho^{-1} x$ and $y \mapsto y$.

We prove now that this condition is necessary.

Notation. For $q \in k^{*}$, and $a \in k[h]$, let $A=A_{q}(a)$ be the GWA $k[h]\left(\sigma_{q}, a\right)$. We fix for all this paragraph $q \in k^{*}$ not a root of unity, and we assume $\operatorname{deg}(a) \geq 1$.

Recall that $A$ is $\mathbb{Z}$-graded by $\operatorname{deg}(h)=0, \operatorname{deg}(x)=1$, and $\operatorname{deg}(y)=-1$ (see [3]), so that

$$
A=\bigoplus_{n>0} k[h] y^{n} \oplus k[h] \oplus \bigoplus_{n>0} k[h] x^{n} .
$$

The following Lemma is the generalization to arbitrary degree of [2], Lemma 4.5.

Lemma 2.2.3 1. Suppose that $a \in k[h]$ is not a monomial. Then the normalizing elements of $A$ are the monomials $\alpha h^{n}$, with $\alpha \in k$ and $n \in \mathbb{N}$.

2. Suppose that $a \in k[h]$ is a monomial. Then the normalizing elements of $A$ are the monomials $\alpha x^{p} h^{n}$, with $\alpha \in k$ and $p, n \in \mathbb{N}$, and $\alpha y^{p} h^{n}$, with $\alpha \in k$ and $p, n \in \mathbb{N}$.

Proof. Let $d \in A$ be a normalizing element. Set $T_{i}=k[h] x^{i}$ if $i \geq 0$ and $T_{i}=k[h] y^{-i}$ if $i<0$, so that $A=\oplus_{n \in \mathbb{Z}} T_{n}$, and write $d=\sum_{i=i_{1}}^{i_{2}} \tilde{d}_{i}$, with $i_{1} \leq$ $i_{2} \in \mathbb{Z}$, and $\tilde{d}_{i} \in T_{i}$. By hypothesis, there exists $s \in A$ such that $h d=d s$. Write again $s=\sum_{j=j_{1}}^{j_{2}} \tilde{s}_{j}$, with $\tilde{s}_{j} \in T_{j}$. Necessarily $h d \in \oplus_{i=i_{1}}^{i_{2}} T_{i}$, so we must have 
$s=\tilde{s}_{0} \in k[h]$. Then $h d=d s$ implies $h \tilde{d}_{i_{1}}=\tilde{d}_{i_{1}} \tilde{s}_{0}$ and $h \tilde{d}_{i_{2}}=\tilde{d}_{i_{2}} \tilde{s}_{0}$. Since $h \tilde{d}_{i}=q^{-i} \tilde{d}_{i} h$ and $q$ is not a root of unity we get $i_{1}=i_{2}$, i.e. $d \in T_{i_{1}}$, and $\tilde{s}_{0}=q^{-i_{1}} h$.

Assume $i_{1}<0$, and put $\iota=-i_{1}=\left|i_{1}\right|$, so that $d=d(h) y^{\iota}$, and $\iota \geq 1$. There exists $t \in A$ such that $x d=d t$. Once again the grading of $A$ implies that $t \in T_{1}$, and we denote $t=t(h) x$. Then

$$
x d=x d(h) y^{\iota}=d(q h)(x y) y^{\iota-1}=d(q h) a(q h) y^{\iota-1} .
$$

Similarly,

$$
d t=d(h) y^{\iota} t(h) x=d(h) t\left(q^{-\iota} h\right) y^{\iota-1} a(h)=d(h) t\left(q^{-\iota} h\right) a\left(q^{1-\iota} h\right) y^{\iota-1} .
$$

Now looking at the degree in $h$ of these two elements of $T_{i_{1}+1}$ we get $t(h)=$ $t_{0} \in k^{*}$ and $d(q h) a(q h)=t_{0} d(h) a\left(q^{1-\iota} h\right)$. Write $a(h)=\sum_{i=n_{1}}^{n} a_{i} h^{i}$, and $d(h)=$ $\sum_{i=m_{1}}^{m} d_{i} h^{i}$, with $0 \leq m_{1} \leq m, 0 \leq n_{1} \leq n$ (we have $n_{1}<n$ if $a$ is supposed not to be a monomial), and $a_{n_{1}}, a_{n}, d_{m_{1}}, d_{m} \in k^{*}$. Identifying the term of highest degree in $d(q h) a(q h)$ and $t_{0} d(h) a\left(q^{1-\iota} h\right)$ we get

$$
q^{m} d_{m} q^{n} a_{n} h^{m+n}=t_{0} d_{m} q^{n(1-\iota)} a_{n} h^{m+n}
$$

so $t_{0}=q^{m+n \iota}$. Then identifying the term of lowest degree we get

$$
q^{m_{1}} d_{m_{1}} q^{n_{1}} a_{n_{1}} h^{m_{1}+n_{1}}=q^{m+n \iota} d_{m_{1}} q^{n_{1}(1-\iota)} a_{n_{1}} h^{m_{1}+n_{1}}
$$

so $m_{1}=m+\left(n-n_{1}\right) \iota$.

Suppose first that $a$ is not a monomial. Then we get a contradiction since $n_{1}<n$. This proves that $i_{1} \geq 0$. Symmetrizing the roles of $x$ and $y$, one gets in an analogous way $i_{1} \leq 0$, and finally $i_{1}=0$, and $d=d(h) \in k[h]$. Then the equality $x d=t x$ becomes $d(q h)=d(h) t(h)$. So $t(h)=t_{0} \in k^{*}$, and $d(h)$ is homogeneous, i.e. it is a monomial.

Now if $a$ is a monomial we have $n_{1}=n$, so $m_{1}=m$, and $d(h)=d_{m} h^{m}$, i.e. $d=$ $d_{m} h^{m} y^{\iota}$. In the same way if $i_{1}>0$ then $d=d_{m} h^{m} x^{i_{1}}$. At last, if $d=d(h) \in k[h]$ is normalizing then it has to be a monomial.

To end the proof, we just have to check that the announced elements are normalizing, which is easily done since $\sigma=\sigma_{q}$.

Remark. Suppose $a=\alpha h^{m}$. Then $A_{q}(a)$ is linearly spanned by its normalizing elements, and can never be isomorphic to a $A_{q}\left(a_{2}\right)$, with $a_{2}$ not a monomial in $k[h]$.

When both $a_{1}$ and $a_{2}$ are monomials, then $A_{q}\left(a_{1}\right)$ and $A_{q}\left(a_{2}\right)$ are isomorphic if and only if $\operatorname{deg}\left(a_{1}\right)=\operatorname{deg}\left(a_{2}\right)$ (this follows for instance from the computation of the Hochschild homology of $A_{q}(a)$, see [9]).

This remark together with the previous results give rise to the following Theorem. 
Theorem 2.2.4 Set $q \in k^{*}$ not a root of unity, and $a_{1}, a_{2} \in k[h]$ are non-constant polynomials. Then there is an isomorphism $\Phi$ from the quantum $G W A A_{q}\left(a_{1}\right)$ onto $A_{q}\left(a_{2}\right)$ if and only if there exist $\alpha, \rho \in k^{*}$ such that $a_{2}(h)=\rho a_{1}(\alpha h)$.

Proof. We saw in proposition 2.2.2 that the condition is sufficient. For the converse, by the remark above we only have to consider the case where neither $a_{1}$ nor $a_{2}$ is a monomial. Denote $h, x, y$ the generators of $A_{q}\left(a_{1}\right)$, and $h^{\prime}, x^{\prime}, y^{\prime}$ the generators of $A_{q}\left(a_{2}\right)$. The isomorphism $\Phi$ maps the normalizing elements of $A_{q}\left(a_{1}\right)$ onto the normalizing elements of $A_{q}\left(a_{2}\right)$. So one necessarily has $\Phi(h)=\alpha h^{\prime}$, with $\alpha \in k^{*}$. Now from the equality $x h=q h x$ in $A_{q}\left(a_{1}\right)$ we deduce $\Phi(x) \alpha h^{\prime}=q \alpha h^{\prime} \Phi(x)$ in $A_{q}\left(a_{2}\right)$. Just like in the proof of Lemma 2.2.3 thanks to the grading of $A_{q}\left(a_{2}\right)$ this equality implies that $\Phi(x)=d\left(h^{\prime}\right) x^{\prime}$, with $d\left(h^{\prime}\right) \in k\left[h^{\prime}\right]$. Considering $\Phi^{-1}$ instead of $\Phi$, one gets in the same way that $\Phi^{-1}\left(x^{\prime}\right)=e(h) x$. So $x=\Phi^{-1}(\Phi(x))=$ $d\left(\alpha^{-1} h\right) e(h) x$ implies $d(h)=\beta \in k^{*}$, and finally $\Phi(x)=\beta x^{\prime}$. Similarly one proves $\Phi(y)=\gamma y^{\prime}$, with $\gamma \in k^{*}$. Then in $A_{q}\left(a_{2}\right)$ one has

$$
a_{1}\left(\alpha h^{\prime}\right)=a_{1}(\Phi(h))=\Phi\left(a_{1}(h)\right)=\Phi(y x)=\gamma y^{\prime} \beta x^{\prime}=\gamma \beta a_{2}\left(h^{\prime}\right),
$$

and we are done.

Remark. By analogy with corollary 2.1.6 one can write in the non-monomial case a multiplicative condition on the roots of $a_{1}$ and $a_{2}$ equivalent to the isomorphism of $A_{q}\left(a_{1}\right)$ and $A_{q}\left(a_{2}\right)$.

Example. After the Theorem we can see that $D_{q, \lambda} \cong D_{q, \lambda^{\prime}}$ if and only if $\lambda^{2}=\lambda^{\prime 2}$.

From the proof of this theorem one deduces easily the automorphism group of $A_{q}(a)$ in the following way.

Theorem 2.2.5 Suppose $a \in k[h]$ is non-monomial. Denote $a=\sum_{i=i_{0}}^{n} a_{i} h^{i}$, with $a_{i} \in k$ and $a_{i_{0}} a_{n} \neq 0$, and define $p=\operatorname{gcd}\left(|i-j|, i, j \in \mathbb{N}, i \neq j, a_{i} a_{j} \neq 0\right)$. Then Aut $A_{q}(a) \simeq(\mathbb{Z} / p \mathbb{Z}) \times k^{*}$.

More precisely, any automorphism of $A_{q}(a)$ is of the form

$$
x \mapsto \alpha x, y \mapsto \gamma^{i_{0}} \alpha^{-1} y ; h \mapsto \gamma h,
$$

with $\alpha \in k^{*}$ and $\gamma$ a pth-root of unity.

Proof. From the proof of theorem 2.2.4 we can deduce that in the nonmonomial case, any automorphism $\sigma$ of $A_{q}(a)$ is defined by $\sigma(x)=\alpha x, \sigma(y)=\beta y$, $\sigma(h)=\gamma h$, with $\alpha, \beta, \gamma \in k^{*}$. Choose then $i>j$ such that $a_{i} a_{j} \neq 0$, then from $\sigma(y) \sigma(x)=a(\sigma(h))$ one gets $\gamma^{i-j}=1$. Then one deduces (for instance using Euclide algorithm) that $\gamma^{p}=1$. Looking at the term of lowest degree in $h$ in these polynomials one gets $\beta=\gamma^{i_{0}} \alpha^{-1}$, where $i_{0}$ is the valuation of polynomial $a(h)$. So $\sigma(x)=\alpha x, \sigma(h)=\gamma h$ and $\sigma(y)=\gamma^{i_{0}} \alpha^{-1} y$.

Conversely, given $\omega$ a pth-root of unity in $k^{*}$ and $\alpha \in k^{*}$, one checks easily that $x \mapsto \alpha x, y \mapsto \omega^{i_{0}} \alpha^{-1}$ and $h \mapsto \omega h$ defines an isomorphism of $A_{q}(a)$. 
Remark. The previous theorem generalizes the computation of the automorphism group of $D_{q, \lambda}$ made in [2], Theorem 4.6.

In the monomial case, the proof is rather similar to the previous one, but we have first to check that any automorphism of $A_{q}(k)$ must send $h$ to $\gamma h$, with $\gamma \in k^{*}$. So assume $a=a_{n} h^{n}$, with $n \geq 1$, and $a_{n} \in k^{*}$.

Proposition 2.2.6 Let $\sigma \in \operatorname{Aut}\left(A_{q}(a)\right)$. Then there exist $\alpha, \gamma \in k^{*}$ such that $\sigma(x)=\alpha x, \sigma(h)=\gamma h$ and $\sigma(y)=\gamma^{n} \alpha^{-1} y$.

Proof. Thanks to Lemma 2.2.3 we have

$$
\sigma(x)=\alpha h^{m_{x}} x^{n_{x}} y^{p_{x}}, \sigma(y)=\beta h^{m_{y}} x^{n_{y}} y^{p_{y}}, \sigma(h)=\gamma h^{m_{h}} x^{n_{h}} y^{p_{h}},
$$

with $\alpha, \beta, \gamma \in k^{*}$, and $n_{x} p_{x}=n_{y} p_{y}=n_{h} p_{h}=0$. From $\sigma(y) \sigma(x)=a_{n} \sigma(h)^{n}$ we get that $n_{x}=n_{y}=0 \Rightarrow n_{h}=0$, and $p_{x}=p_{y}=0 \Rightarrow p_{h}=0$. Because $\sigma$ is surjective we must have $n_{x}+n_{y}+n_{h} \neq 0$ and $p_{x}+p_{y}+p_{h} \neq 0$, so we only have two possibilities : $n_{x} p_{y} \neq 0$ or $n_{y} p_{x} \neq 0$.

Consider firstly the case $n_{x} p_{y} \neq 0$, and assume that $p_{y} \geq n_{x}$. Then

$$
\begin{gathered}
\sigma(y) \sigma(x)=\beta \alpha h^{m_{y}} y^{p_{y}} h^{m_{x}} x^{n_{x}}=\beta \alpha q^{*} h^{m_{x}+m_{y}} y^{p_{y}-n_{x}} y^{n_{x}} x^{n_{x}} \\
=a_{n}^{n_{x}} \beta \alpha q^{*} h^{m_{x}+m_{y}+n n_{x}} y^{p_{y}-n_{x}},
\end{gathered}
$$

where $*$ represents some integer.

So $\sigma(h)=\gamma h^{m_{h}} y^{p_{h}}$, and $a \sigma(h)^{n}=a \gamma^{n} q^{*} h^{n m_{h}} y^{n p_{h}}$. We have now the following equalities :

$$
\left\{\begin{array}{c}
m_{x}+m_{y}+n n_{x}=n m_{h} \\
p_{y}-n_{x}=n p_{h}
\end{array}\right.
$$

Now, from $y h=q^{-1} h y$ we get $\sigma(y) \sigma(h)=q^{-1} \sigma(h) \sigma(y)$, that is

$$
\beta \gamma q^{-p_{y} m_{h}} h^{m_{y}+m_{h}} y^{p_{y}+p_{h}}=\gamma \beta q^{-1-p_{h} m_{y}} h^{m_{h}+m_{y}} y^{p_{h}+p_{y}} .
$$

Because $q$ is not a root of unity we get $-p_{y} m_{h}=-1-p_{h} m_{y}$. Replacing $p_{y}$ by its expression above we get $-\left(n p_{h}+n_{x}\right) m_{h}=-1-p_{h} m_{y}$, that is $p_{h}\left(n m_{h}-m_{y}\right)+$ $n_{x} m_{h}=1$. Using the first equality above we finally have $p_{h}\left(m_{x}+n n_{x}\right)+n_{x} m_{h}=1$. For $n \geq 2$ we must have $p_{h}=0$ and $n_{x}=m_{h}=1$. From $m_{x}+m_{y}+n n_{x}=n m_{h}$ we get then $m_{x}=m_{y}=0$, and from $p_{y}-n_{x}=n p_{h}$ we get $p_{y}=1$.

For $n=1$ if $p_{h}=0$ we get the same conclusion, elsewhere we have $p_{h}=n_{x}=1$ and $m_{x}=m_{h}=0$. But then $m_{x}+m_{y}+n n_{x}=n m_{h}$ is impossible, so we get a contradiction. Finally for any $n \geq 1$ we have $\sigma(x)=\alpha x, \sigma(y)=\beta y$ and $\sigma(h)=\gamma h$.

Assuming that $p_{y}<n_{x}$, analoguous computations lead to $p_{y}=n_{x}=1$, a contradiction.

The second case to consider is $n_{y} p_{x}=0$. The reader may check that once again the same type of computations lead to expressions of the form "sum of nonnegative integers $=-1$ ", a contradiction.

At last, because $\sigma(y) \sigma(x)=a \sigma(h)^{n}$, we get $\alpha \beta=\gamma^{n}$.

Then one easily obtains the following. 
Corollary 2.2.7 Suppose $a(h)=a_{n} h^{n}$ is monomial. Then Aut $A_{q}(a) \simeq k^{*} \times k^{*}$. More precisely, any automorphism of $A_{q}(a)$ is of the form

$$
x \mapsto \alpha x, y \mapsto \gamma^{n} \alpha^{-1} y ; h \mapsto \gamma h,
$$

with $\alpha, \gamma \in k^{*}$.

The last part of this section is devoted to some application to Smith algebras.

\subsection{Smith algebras.}

Let $q \in k^{*}$ not a root of unity, and $\sigma=\sigma_{q}$. We consider the $k$-algebra $R(f)$ freely generated by $x, y, h$ modulo relations

$$
x h=\sigma(h) x, \quad h y=y \sigma(h), \quad x y-y x=f(h) .
$$

where $f(0)=0$. This hypothesis ensures that there exists a polynomial $a \in k[h]$ such that $f(h)=a(\sigma(h))-a(h)$. The algebra $R(f)$ has been studied by S. Smith in [16. Note that the polynomial $a$ is determined up to its constant term.

Our interest in this algebra comes from the fact that $A_{q}(a)$ is the quotient of $R(f)$ by the two sided ideal generated by $\Omega=y x-a(h)$. In particular, $R(f)$ has a filtration, setting $d g(x)=d g(y)=n$ and $d g(h)=2$ (where $n$ is the degree of the polynomial $f$ ), inducing a filtration on $A_{q}(a)$, and it is clear that the associated graded algebra of $R(f)$ is the quantum affine space $k<x, y, h \mid x y=y x ; x h=$ $q h x ; y h=q^{-1} h y>$.

Remark. One can easily check that $\operatorname{Frac}(R(f))$ is the fraction field of the $k$ algebra generated over the centre $k[\Omega]$ by $x$ and $h$ satisfying $x h=q h x$.

We will prove the following Theorem, generalizing Theorem 5.4 of [5].

Theorem 2.3.1 Suppose that we have two Smith algebras, $R\left(f_{1}\right)$ and $R\left(f_{2}\right)$, with the above notations. Then there is an isomorphism $\Phi$ between them if and only if there exist $\alpha \in k, \beta, \rho \in k^{*}$ such that $a_{1}(h)=\rho a_{2}(\beta h)+\alpha$.

Proof. It is easy to show that the centre of $R\left(f_{i}\right)$ is generated by $\Omega_{i}$. The isomorphism $\Phi$ then sends $\Omega_{1}$ to $\beta \Omega_{2}+\alpha$, for some $\beta \in k^{*}$ and $\alpha \in k$. So $\Phi$ induces an isomorphism between $A\left(a_{1}-\alpha\right)$ and $A\left(a_{2}\right)$. We can now conclude thanks to Theorem 2.2.4.

Example. In his article [18, Witten introduces a 7-parameter deformation of the universal enveloping algebra $\mathcal{U}\left(s l_{2}\right)$. This deformation is a unital associative algebra over $\mathbb{C}$, with generators $x, y, z$ satisfying relations

$$
\begin{aligned}
x z & =\left(\epsilon_{2}+\epsilon_{1} z\right) x, \\
z y & =y\left(\epsilon_{4}+\epsilon_{3} z\right), \\
y x-\epsilon_{5} x y & =\epsilon_{6} z^{2}+\epsilon_{7} z,
\end{aligned}
$$


where $\epsilon=\left(\epsilon_{1}, \ldots, \epsilon_{7}\right) \in \mathbb{C}^{7}$. This algebra is denoted $\mathcal{M}(\epsilon)$.

If

$$
\epsilon_{1}=\epsilon_{3} \notin\{0,-1,1\}, \epsilon_{2}=\epsilon_{4}, \epsilon_{5}=1 \text { and } \epsilon_{7}=\frac{2 \epsilon_{6} \epsilon_{1} \epsilon_{2}}{\left(\epsilon_{1}^{2}-1\right)},
$$

then $\mathcal{M}(\epsilon)$ is isomorphic to the Smith algebra with generators $x, y$ over $\mathbb{C}[z]$, $\sigma(z)=\epsilon_{2}+\epsilon_{1} z$ and $f(z)=a(\sigma(z))-a(z)=\epsilon_{6} z^{2}+\epsilon_{7} z$, where $a(z)$ is defined up to a constant by

$$
a(z)=\frac{\epsilon_{6}}{\epsilon_{1}^{2}-1} z^{2}-\frac{\epsilon_{6} \epsilon_{2}^{2}}{\epsilon_{1}\left(\epsilon_{1}^{2}-1\right)} z+a_{0} .
$$

By using theorem 2.3.1. this algebra is isomorphic to the Smith algebra with defining polynomial $a(z)=z^{2}-\left(\epsilon_{2}^{2} / \epsilon_{1}\right) z$.

If $\epsilon_{1}=1$, then $a(z)=\left(\epsilon_{7} /\left(2 \epsilon_{2}\right)\right) z^{2}-\left(\left(\epsilon_{7} \epsilon_{2}\right) / 2\right) z+a_{0}$, for $\epsilon_{2} \neq 0$, and the algebra is isomorphic to the Smith algebra with defining polynomial $\tilde{a}(z)=z^{2}-z$. In this situation $\epsilon_{6}$ must be 0 .

If $\epsilon_{1}=-1, \epsilon_{6}=0$ and $\epsilon_{2} \neq 0,1$ then we can choose $a(z)=z^{2}-z$.

Conversely, any Smith algebra with $\operatorname{deg} f(z)=2$ and $f(0)=0$ is isomorphic to a Witten deformation algebra $\mathcal{M}(\epsilon)$ whose parameters satisfy (3).

Also, a deformation algebra has a filtration. Le Bruyn (cf. [12] and [13]) studied the algebras $\mathcal{M}(\epsilon)$ whose associated graded algebra are Auslander-regular. He proved that they determine a 3-parameter family of Witten deformation algebras, with defining relations:

$$
\begin{aligned}
x z & =(1+\alpha z) x, \\
z y & =y(1+\alpha z), \\
y x-\gamma x y & =\beta z^{2}+z .
\end{aligned}
$$

When $\gamma=1$ this algebra is called "conformal $\mathfrak{s} l_{2}$-algebra". When $\alpha \neq \pm 1$, it is isomorphic to the Smith algebra over $\mathbb{C}[z]$ with generators $x$ and $y, \sigma(z)=$ $1+\alpha z$, and $a(z)=\left(\beta /\left(\alpha^{2}-1\right)\right) z^{2}+\left(\beta /\left(\alpha\left(1-\alpha^{2}\right)\right)\right) z+a_{0}$. We can choose, up to isomorphism, $a(z)=z^{2}-z / \alpha$.

Remarks similar to those ones of the above example for down-up algebras instead of Smith algebras can be found in [7].

\section{Morita equivalence.}

\subsection{A necessary condition.}

The following Lemma provides a necessary condition for Morita equivalence of two $k$-algebras.

Lemma 3.1.1 Let $A$ and $B$ be two k-algebras, and assume they are noetherian domains. If they are Morita equivalent, then their fraction fields Frac $(A)$ and $\operatorname{Frac}(B)$ are isomorphic. 
Proof. The rings $A$ and $B$ are Morita equivalent, that is, the category of (for example) left $A$-modules, $A-M o d$ is equivalent to $B-M o d$. We use now the Example 10.3.16 of [17]. Let $\Sigma_{A}$ and $\Sigma_{B}$ be the collections of morphisms $f \in A-\operatorname{Mod}$ (resp. in $B-M o d$ ) that become isomorphims after tensoring over $A$ (resp. $B)$ by $\operatorname{id}_{\operatorname{Frac}(A)}$ (resp. $\left.\operatorname{id}_{\operatorname{Frac}(B)}\right) . \quad \Sigma_{A}$ and $\Sigma_{B}$ are multiplicative systems, and the category $\operatorname{Frac}(A)-\operatorname{Mod}$ is localizing, since the canonical map $M \rightarrow \operatorname{Frac}(A) \otimes_{A} M$ belongs to $\Sigma_{A}$, for all $M \in A-M o d$.

Since $\Sigma_{A} \cap \operatorname{Frac}(A)-\operatorname{Mod}$ consists of isomorphims, we have that $\operatorname{Frac}(A)-\operatorname{Mod} \cong$ $\Sigma_{A}^{-1}(A-M o d)$. Similar facts hold for $B$. Also, $f \in \Sigma_{A}$ if and only if its image under the equivalence functor is in $\Sigma_{B}$.

As a consequence $\operatorname{Frac}(A)$ and $\operatorname{Frac}(B)$ are Morita equivalent rings, but since they are division rings, the respective endomorphism rings are simple, and then they are isomorphic.

Remarks • One may only suppose that one of the algebras is noetherian, because this is a Morita-invariant property (but not the property of being an integral domain).

- This Lemma separates up to Morita equivalence the Weyl algebra $A_{n}(k)$ from algebras $S_{n, n}^{\Lambda}(k)$ of twisted differential operators on an affine quantum space studied in [15], where these algebras where shown to have the same Hochschild homology and cohomology.

Now we apply this Lemma to GWA. Note that simplicity is a Morita invariant property too, and that a quantum GWA $A_{q}(a)=k[h]\left(\sigma_{q}, a\right)$ is never simple because $h$ is normal and generates a non-trivial two-sided ideal. To have a simple algebra one may localize by $h$, and consider $A_{q}^{\prime}(a)=k\left[h^{ \pm 1}\right]\left(\sigma_{q}, a\right)$. Then one has the following criteria for simplicity. Note that a Laurent polynomial $a(h)$ can always be uniquely written as $a(h)=h^{m} \tilde{a}(h)$, with $m \in \mathbb{Z}, \tilde{a}(h) \in k[h]$ and $\tilde{a}(0) \neq 0$. By roots of a Laurent polynomial $a$ we mean the (non-zero) roots of the associated polynomial $\tilde{a}$.

Proposition 3.1.2 1. The classical $G W A A(a)=k[h]\left(\sigma_{c l}, a\right)$ is simple if and only if the polynomial a only has simple roots, and for any two distinct roots $\alpha$ and $\beta$ of $a$, then $\alpha-\beta \notin \mathbb{Z}$.

2. The algebra $A_{q}^{\prime}(a)=k\left[h^{ \pm 1}\right]\left(\sigma_{q}, a\right)$ is simple if and only if $q$ is not a root of unity, the Laurent polynomial a only has simple roots, and for any two distinct roots $\alpha$ and $\beta$ of a, there exist no $m \in \mathbb{Z}$ such that $\alpha=q^{m} \beta$.

Proof. This follows from proposition 2 and corollary 2 of [4].

It is clear that, just like in proposition 2.1.1 the algebra $A_{q}^{\prime}(a)=k\left[h^{ \pm 1}\right]\left(\sigma_{q}, a\right)$ has fraction field the quantum skew-field $k_{q}(u, v)$. So applying Lemma 3.1.1 together with the results of the first section we obtain the following.

Proposition 3.1.3 1. A classical $G W A A\left(a_{1}\right)=k[h]\left(\sigma_{c l}, a_{1}\right)$ is never Morita equivalent to a quantum GWA. 
2. If $A_{q_{1}}\left(a_{1}\right)$ and $A_{q_{2}}\left(a_{2}\right)$ are two Morita equivalent quantum $G W A$, then $\left\langle q_{2}\right\rangle=$ $\left\langle q_{1}\right\rangle$ in $k^{*}$.

3. If $A_{q_{1}}^{\prime}\left(a_{1}\right)$ and $A_{q_{2}}^{\prime}\left(a_{2}\right)$ are two Morita equivalent simple quantum $G W A$, then $q_{2}=q_{1}^{ \pm 1}$ in $k^{*}$.

Proof. 1. If $A\left(a_{1}\right)=k[h]\left(\sigma_{c l}, a_{1}\right)$ is Morita equivalent to a noetherian domain $B$, then $\operatorname{Frac}\left(A\left(a_{1}\right)\right)=\mathcal{D}_{1}(k)$ is isomorphic to $\operatorname{Frac}(B)$ by Lemma 3.1.1 and by corollary 2.1.4 $\operatorname{Frac}(B)$ cannot be a quantum skew-field, so $B$ cannot be a quantum GWA.

Points 2 and 3 are proved in an analogous way, using the invariant $G$ defined in 2.1.2 For the conclusion of point 3, note that in the simple case $q_{1}$ and $q_{2}$ are not roots of unity.

Remarks. - In the classical case, the Hochschild homology and cohomology are computed in [8], giving necessary conditions for Morita equivalence linked to the degree of the polynomials $a_{1}$ and $a_{2}$.

- As already mentioned after corollary 2.1.4 the GWA $k\left[h^{ \pm 1}\right](\sigma, a(h))$ is isomorphic to $k\left[h^{ \pm 1}\right]\left(\sigma^{-1}, a(\sigma(h))\right)$ by $(x, y) \mapsto(y, x)$. So in point 3 one may conclude that up to isomorphism we have $q_{2}=q_{1}$.

\subsection{Remarks about the classification problem.}

\section{The classical case.}

For $a \in k[h]$, let $A(a)$ denote the GWA $k[h]\left(\sigma_{c l}, a\right)$.

- If $\operatorname{deg}(a)=1$, then $A(a)$ is isomorphic to the Weyl algebra $A_{1}(k)$.

- If $\operatorname{deg}(a)=2$, then $A(a)$ is a prime quotient of the enveloping algebra $\mathcal{U}\left(s l_{2}\right)$, and the classification up to Morita equivalence is completely solved by Hodges in [10] by means of the following Theorem.

Theorem 3.2.1 Set $a_{1}, a_{2} \in k[h]$ two polynomials of degree 2 with distinct roots respectively $\alpha_{1} \neq \alpha_{2}$ and $\alpha_{1}^{\prime} \neq \alpha_{2}^{\prime}$. Then the $G W A A\left(a_{1}\right)$ and $A\left(a_{2}\right)$ are Moritaequivalent if and only if there exist $\epsilon \in\{-1,1\}$ and $m \in \mathbb{Z}$ such that $\alpha_{1}-\alpha_{2}=$ $\epsilon\left(\alpha_{1}^{\prime}-\alpha_{2}^{\prime}\right)+m$.

Proof. This is just a rephrasing in terms of roots of the polynomials of [10, Theorem 5 .

- If $\operatorname{deg}(a) \geq 3$, we can prove the following sufficient condition.

Proposition 3.2.2 Set $a_{1}, a_{2} \in k[h]$ two polynomials of degree $n \geq 3$ satisfying the simplicity criterium of proposition [3.1.2(1), with distinct roots respectively $\alpha_{1}, \ldots, \alpha_{n}$ and $\alpha_{1}^{\prime}, \ldots, \alpha_{n}^{\prime}$. Suppose that there exist $\tau \in \mathcal{S}_{n}$ and $\left(m_{1}, \ldots, m_{n}\right) \in \mathbb{Z}^{n}$ such that $\alpha_{i}=\alpha_{\tau(i)}^{\prime}+m_{i}$ for all $1 \leq i \leq n$. Then the $G W A A\left(a_{1}\right)$ and $A\left(a_{2}\right)$ are Morita-equivalent. 
Proof. This is a direct consequence of Lemma 2.4 and Theorem 2.3 of [1].

This condition is exactly the analogous in degree $n$ of the condition of Theorem 3.2.1 So, as already noted by Hodges, it would be interesting to know a necessary condition in any degree. Since the condition appearing in the above proposition is written in terms of the roots of the polynomials, so one may compare it with corollary 2.1.6

\section{The quantum case.}

We consider the non root of unity case. At this point, we have some necessary conditions, first by proposition 3.1 .3 for $A_{q_{1}}\left(a_{1}\right)$ and $A_{q_{2}}\left(a_{2}\right)$ to be Morita equivalent we must have $q_{2}=q_{1}^{ \pm 1}$. Moreover the degree of the polynomial used to define a GWA is linked to its Hochschild homology, computed by Farinati, Solotar and Suárez-Álvarez in [9]. Then if $A_{q}\left(a_{1}\right)$ and $A_{q}\left(a_{2}\right)$ are Morita equivalent one must also have that $\operatorname{deg}\left(a_{1}\right)=\operatorname{deg}\left(a_{2}\right)$ and the same for the polynomials $\operatorname{gcd}\left(a_{1}, a_{1}^{\prime}\right)$ and $\operatorname{gcd}\left(a_{2}, a_{2}^{\prime}\right)$. We can say too that the sufficient conditions mentioned in proposition 3.2.2 are still true in the quantum case, replacing in Hodges' proofs (11], Lemma 2.4 and Theorem 2.3) $\sigma_{c l}$ by $\sigma_{q}$. Then the condition $\alpha_{i}=\alpha_{\tau(i)}^{\prime}+m_{i}$ becomes $\alpha_{i}=q^{m_{i}} \alpha_{\tau(i)}^{\prime}$.

\section{References}

[1] J. Alev, F. Dumas, Sur le corps de fractions de certaines algèbres quantiques, J. Algebra 170 (1994), no. 1, 229-265.

[2] J. Alev, F. Dumas, Rigidité des plongements des quotients primitifs minimaux de $U_{q}(\mathfrak{s l}(2))$ dans l'algèbre quantique de Weyl-Hayashi, Nagoya Math. J. 143 (1996), 119-146.

[3] V.V. Bavula, Generalized Weyl algebras and their representations, St. Petersburg Math. J. 4 (1993), no. 1, 71-92.

[4] V.V. Bavula, Description of bilateral ideals in a class of noncommutative rings. I, Ukrainian Math. J. 45 (1993), no. 2, 223-234.

[5] V.V. Bavula, D.A. Jordan, Isomorphism problems and groups of automorphisms for generalized Weyl algebras, Trans. Amer. Math. Soc. 353 (2001), no. 2, 769-794.

[6] V. Bavula, T. Lenagan, Krull dimension of generalized Weyl algebras with noncommutative coefficients, J. of Alg. 235, (2001), no 1, 315-358.

[7] G. Benkart, T. Roby, Down-up algebras, J. of Alg. 209, (1998), 305-344.

[8] M.A. Farinati, A.L. Solotar, M. Suárez-Álvarez, Hochschild homology and cohomology of generalized Weyl algebras, Ann. Inst. Fourier (Grenoble) 53 (2003), no. 2, 465-488. 
[9] M.A. Farinati, A.L. Solotar, M. Suárez-Álvarez, Hochschild homology and cohomology of quantum GWA, preprint.

[10] T.J. Hodges, Morita equivalence of primitive factors of $U(\mathfrak{s l}(2))$, in KazhdanLusztig theory and related topics (Chicago, IL, 1989), Contemp. Math., 139, Amer. Math. Soc., Providence, RI, 1992, 175-179.

[11] T.J. Hodges, Noncommutative deformations of type-A Kleinian singularities, J. Algebra 161 (1993), no. 2, 271-290.

[12] L. Le Bruyn, Two remarks on Witten's quantum enveloping algebra, Comm. Algebra 22 (1994), 865-870.

[13] L. Le Bruyn, Conformal $\mathfrak{s} l_{2}$ enveloping algebras, Comm. Algebra 23 (1995), $1325-1362$.

[14] J.C. MacConnell, J.C. Robson, Noncommutative noetherian rings. Revised edition. Graduate Studies in Mathematics, Vol. 30. American Mathematical Society, Providence, RI, 2001.

[15] L. Richard, Hochschild homology and cohomology of some classical and quantum noncommutative polynomial algebras, J. Pure Appl. Algebra 187 (2004), nos. 1-3, 255-294.

[16] S. Smith, A class of algebras similar to the enveloping algebra of $\mathfrak{s l}_{2}$, Trans. Am. Math. Soc. 322, (1990), no. 1, 285-314.

[17] C.A. Weibel, An introduction to homological algebra, Cambridge Studies in Advanced Mathematics, Vol. 38. Cambridge University Press, Cambridge, 1994.

[18] E. Witten, Quantization of Chern-Simons gauge theory with complex gauge group, Comm. Math. Physics 137 (1991), 29-66. 